SUBJECT AREAS: CANCER MODELS COMPUTATIONAL MODELS COMPUTATIONAL BIOPHYSICS BAYESIAN INFERENCE

SCIENTIFIC REPORTS:

$2: 990$

DOI: $10.1038 /$ srep00990

(2012)

Published:

18 December 2012

Updated: 3 April 2014

\section{CORRIGENDUM: Interplay between distribution of live cells and growth dynamics of solid tumours}

\author{
Edoardo Milotti, Vladislav Vyshemirsky, Michela Sega \& Roberto Chignola
}

There is an error in the definition of priors used in Bayesian analysis of the newly proposed model of tumour growth dynamics in our published paper. Instead of the reported exponential priors for $\lambda$ parameters with a mean of $100 \mu \mathrm{m}$, as defined in equation (15), a mean of $10000 \mu \mathrm{m}$ was used by mistake. This resulted in overly spread model posteriors. All model predictions from erroneous posteriors match the data well, however estimated parameter values are outside of reasonable ranges.

Additionally, the corrected prior, being more restrictive, acts as an additional evidence for the model with constant $\lambda$. As a result, the model with variable $\lambda$ is penalised for being overly complex in model comparison. A model with variable $\lambda$ predicts data equally well, however it has more parameters and a more complex structure. There is no substantial evidence to prefer a model with variable $\lambda$ over the one with constant $\lambda$.

Consequently, some plots and reported figures are incorrect in the published paper. The corrected results do not differ from the original publication in any qualitative manner. All of the original conclusions are still correct considering the updated results.

1. There is a misprint in the equation below figure 1 , instead of

$$
\int_{0}^{x} e^{-(x-s) / \lambda} A \frac{(x-s)^{2}}{x^{2}} d s \approx \frac{3 \lambda}{3 \lambda+[V(x) / A]^{1 / 3}} V(x)
$$

it should read

$$
\int_{0}^{x} e^{-(x-s) / \lambda} 3 A(x-s)^{2} d s \approx \frac{3 \lambda}{3 \lambda+[V(x) / A]^{1 / 3}} V(x)
$$

2. In the legend of Figure 2 “ $\lambda_{0}=48.96 \pm 0.05 \mu \mathrm{m}, \lambda_{1}=77.48 \pm 0.05 \mu \mathrm{m}, \zeta=48.96 \pm 0.05 \mu \mathrm{m}$ " should read “ $\lambda_{0}=48.96 \pm 0.06 \mu \mathrm{m}, \lambda_{1}=77.48 \pm 0.06 \mu \mathrm{m}, \zeta=167.32 \pm 0.06 \mu \mathrm{m}$ ".

3. Figure 3 is affected by the wrong selection of prior described above. Therefore, in the legend of Figure 3 " $\lambda_{c}=$ $15.3 \pm 1.7 \mu \mathrm{m}(9 \mathrm{l}) ; \lambda_{c}=19.3 \pm 1.9 \mu \mathrm{m}$ (U118); $\lambda_{c}=16.2 \pm 1.9 \mu \mathrm{m}$ (MCF7)" should read " $\lambda_{c}=121.0 \pm$ $92.8 \mu \mathrm{m}$ (91); $\lambda_{c}=161 \pm 48.2 \mu \mathrm{m}$ (U118); $\lambda_{c}=152.2 \pm 101.2 \mu \mathrm{m}$ (MCF7)". Moreover figure 3 is incorrect. The correct figure 3 appears below as figure 1 .

4. Figure 4 is also affected by the wrong selection of prior described above. The correct figure 4 appears below as figure 2 .

5. Figure 5 is also affected by the wrong selection of prior described above. The correct figure 5 appears below as figure 3.

6. Finally, the wrong selection of priors affects parts of the text in the section 91 cell line.

a. "(correlation coefficient is 0.996 in this case)" should read "(correlation coefficient is 0.993 in this case)";

b. "At the same time, the observation noise with variance $\sigma^{2}$ considered in both models, was found to be almost independent a posteriori from other model parameters, with the largest correlation coefficient for Gompertz model of 0.07 (correlation of $\sigma$ and $\alpha_{G}$ ), and the largest correlation coefficient for the new model of 0.059 (correlation of $\sigma$ and $\alpha$ ), and the largest correlation coefficient for the new model with variable $\lambda$ of -0.024 (correlation of $\sigma$ and $\delta$ )." should read "At the same time, the observation noise with variance $\sigma^{2}$ considered in both models, was found to be almost independent a posteriori from other model parameters, with the largest correlation coefficient for Gompertz model of 0.01 (correlation of $\sigma$ and $\alpha_{G}$ ), and the largest correlation coefficient for the new model of -0.07 (correlation of $\sigma$ and $\lambda$ ), and the largest correlation coefficient for the new model with variable $\lambda$ of 0.09 (correlation of $\sigma$ and $\zeta$ ).";

c. "The weakest Bayes factor in this sample - for spheroid 31, predictions depicted in the penultimate plot of Figure S5 - qualified the category of evidence support as 'positive' while for every other spheroid the preference for the new model was 'very strong." should read "The weakest Bayes factor in this sample - for 

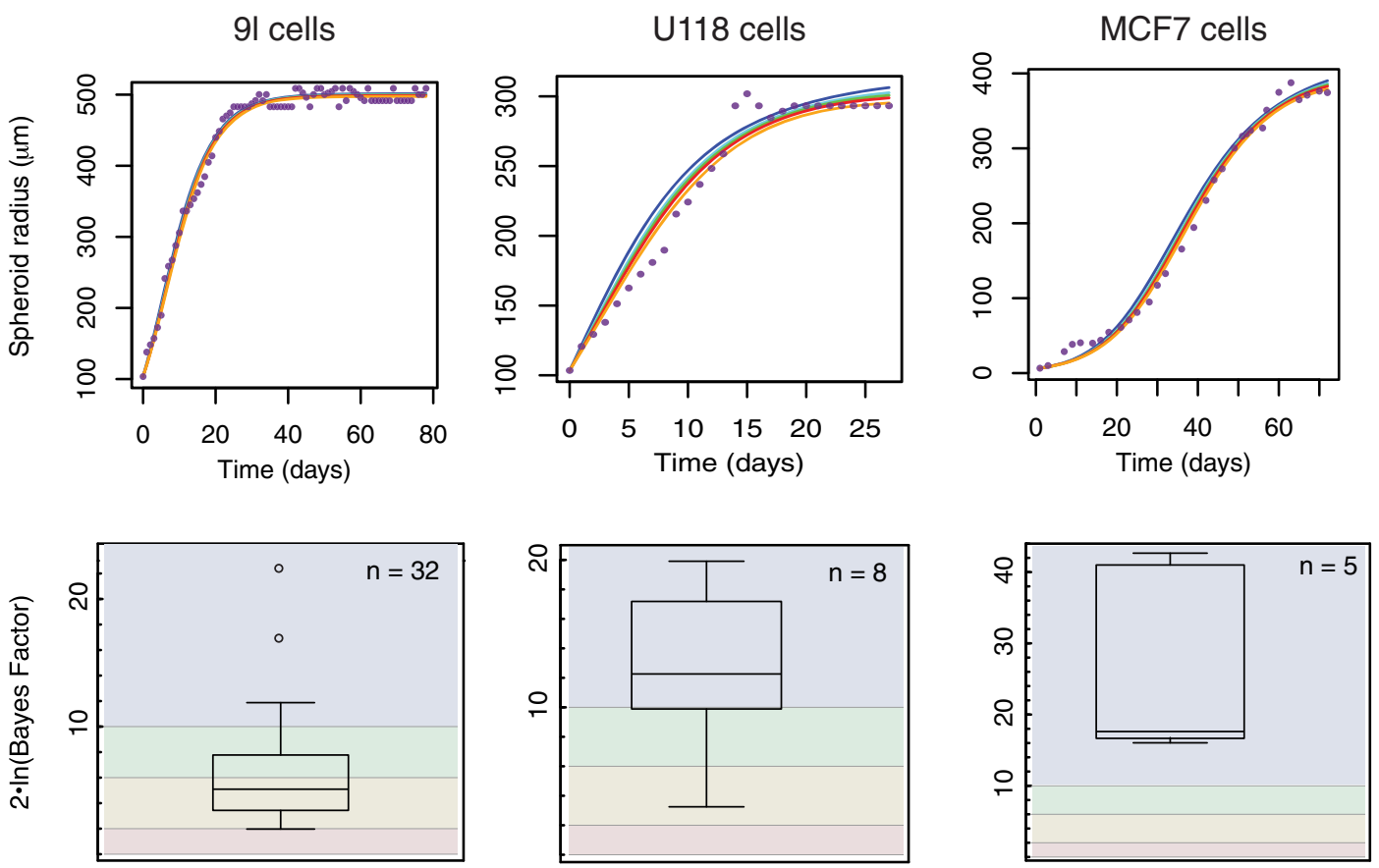

Figure $1 \mid$

spheroid 22, predictions depicted in Figure S5 - qualified the category of evidence support as 'weak' while for every other spheroid the preference for the new model was substantially stronger."

In the Supplementary Information the first two sections are unchanged. All of the figures of section 3, except figure S4, are affected by the change in the prior distribution, and they have been replaced by new ones.

1. the title of section 3 "Results of the Bayesian data analyses" now reads "Results of the Bayesian data analysis"

2. the beginning of section 3 "Here we summarize the results of the Bayesian data analyses ..." now reads "Here we summarize the results of the Bayesian data analysis ..."

3. the caption of figure S10 "Bayes factors computed using the data from 32 spheroids of the 91 cell line. The box plot demonstrates that the new model with variable $\lambda$ is slightly better supported by the data than the one with constant $\lambda$. The categories of evidence support defined in Table S2 are plotted against our results." now reads "Bayes factors computed using

$\mu \mathrm{m}$

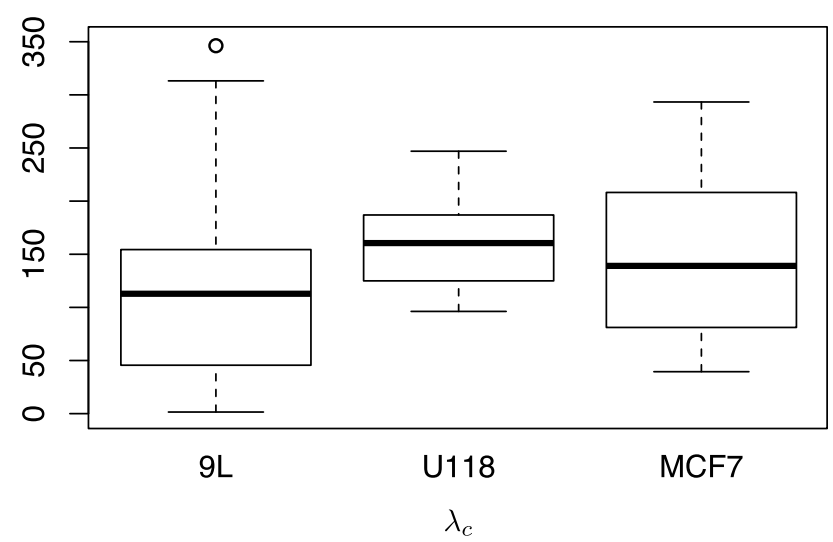

Figure 2 the data from 32 spheroids of the 91 cell line. The box plot demonstrates that the new model with constant $\lambda$ is slightly better supported by the data than the one with variable $\lambda$. This can be justified by increased model complexity for a variable $\lambda$ model. These results show no clear cut preference among the two models. The categories of evidence support defined in Table S2 are plotted against our results."

4. the caption of figure S11 "A posteriori odds of the alternative models given data from 91 cell line demonstrate that new models are significantly preferred to the traditional Gompertz model, while the evidence is not very high to decisively prefer the new model with variable $\lambda$ over the one with constant $\lambda$. ." now reads "A posteriori odds of the alternative models given data from 91 cell line demonstrate that new models are significantly preferred to the traditional Gompertz model, while the evidence is not very high to decisively prefer the new model with constant $\lambda$ over the one with variable $\lambda . "$

5. the caption of figure S16 "Bayes factors computed using the data from 8 spheroids of the U118 cell line. The box plot demonstrates that every single Bayes factor very strongly prefers the new model over the traditional Gompertz model. The categories of evidence support defined in Table S2 are plotted against our results." now reads "Bayes factors computed using the data from 8 spheroids of the U118 cell line. The box plot demonstrates that every single Bayes factor prefers the new model over the traditional Gompertz model. The categories of evidence support defined in Table S2 are plotted against our results."

6. the caption of figure S17 "Bayes factors computed using the data from 8 spheroids of the U118 cell line. The box plot demonstrates that the new model with variable $\lambda$ is slightly better supported by the data than the one with constant $\lambda$. The categories of evidence support defined in Table S2 are plotted against our results." now reads "Bayes factors computed using the data from 8 spheroids of the U118 cell line. The box plot demonstrates that the new model with constant $\lambda$ is better supported by the data than the one with variable $\lambda$. The categories of evidence support defined in Table S2 are plotted against our results" 


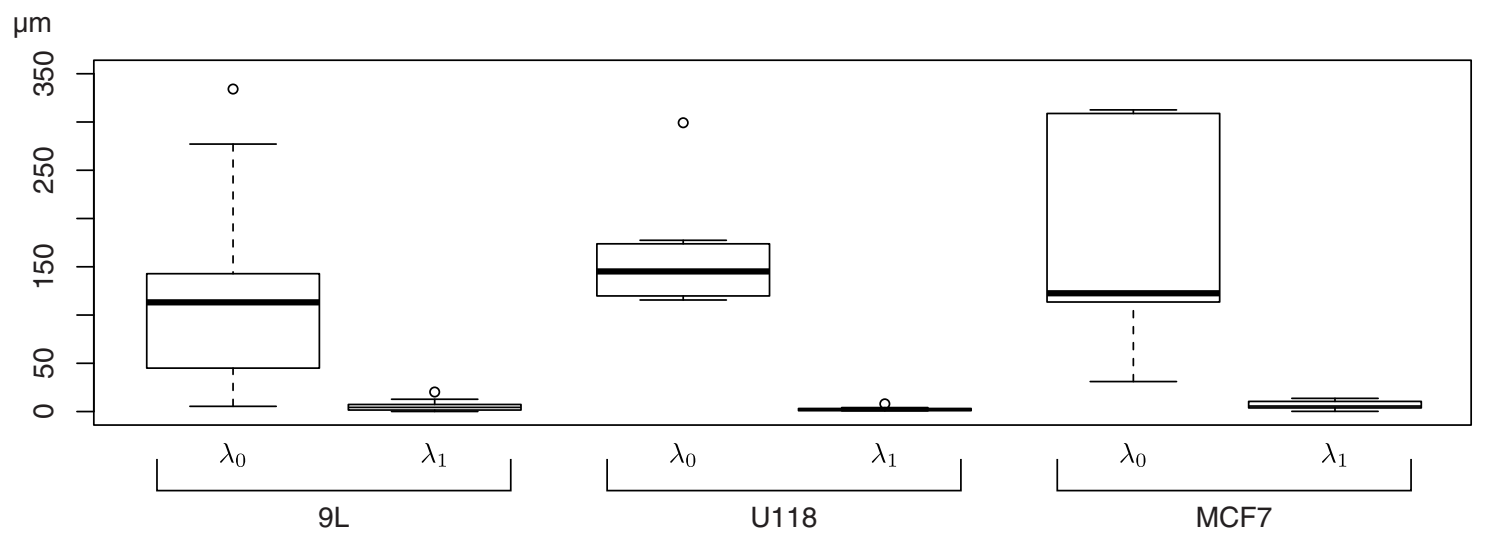

Figure 3

7. the caption of figure S18 "A posteriori odds of the alternative models given data from U118 cell line demonstrate that new models are significantly preferred to the traditional Gompertz model, while the evidence is not very high to decisively prefer the new model with variable $\lambda$ over the one with constant $\lambda . "$ now reads "A posteriori odds of the alternative models given data from U118 cell line demonstrate that the new models are significantly preferred to the traditional Gompertz model, while the evidence is not very high to decisively prefer the new model with constant $\lambda$ over the one with variable $\lambda . "$

8. the caption of figure S24 "Bayes factors computed using the data from 8 spheroids of the MCF7 cell line. The box plot demonstrates that the new model with variable $\lambda$ is better supported by the data than the one with constant $\lambda$. The categories of evidence support defined in Table S2 are plotted against our results." now reads "Bayes factors computed using the data from 8 spheroids of the MCF7 cell line. The box plot demon- strates that the new model with variable $\lambda$ is not better supported by the data than the one with constant $\lambda$. Different data sets support different formulations of the model. The model with variable $\lambda$ is penalised for complexity. The results do not demonstrate a clear cut preference among the new models. The categories of evidence support defined in Table S2 are plotted against our results."

9. the caption of figure S25 "A posteriori odds of the alternative models given data from MCF7 cell line demonstrate that new models are significantly preferred to the traditional Gompertz model, while the evidence is not very high to decisively prefer the new model with variable $\lambda$ over the one with constant $\lambda$. ." now reads "A posteriori odds of the alternative models given data from MCF7 cell line demonstrate that the new models are significantly preferred to the traditional Gompertz model, while the evidence is not very high to decisively prefer either the new model with variable $\lambda$ or the one with constant $\lambda . "$ 